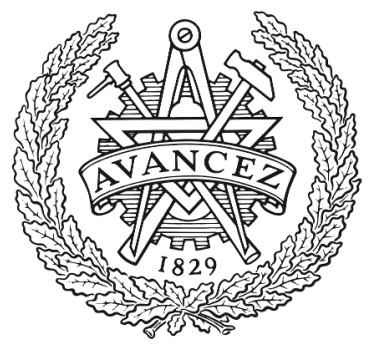

CHALMERS

UNIVERSITY OF TECHNOLOGY

\title{
Multimillijoule terahertz radiation from laser interactions with microplasma waveguides
}

Downloaded from: https://research.chalmers.se, 2023-04-26 11:07 UTC

Citation for the original published paper (version of record):

Hu, K., Yi, L., Fülöp, T. (2021). Multimillijoule terahertz radiation from laser interactions with microplasma waveguides. Plasma Physics and Controlled Fusion, 63(3).

http://dx.doi.org/10.1088/1361-6587/abdcdc

N.B. When citing this work, cite the original published paper. 
PAPER • OPEN ACCESS

Multimillijoule terahertz radiation from laser interactions with microplasma waveguides

To cite this article: Ke Hu et al 2021 Plasma Phys. Control. Fusion 63035028

View the article online for updates and enhancements.

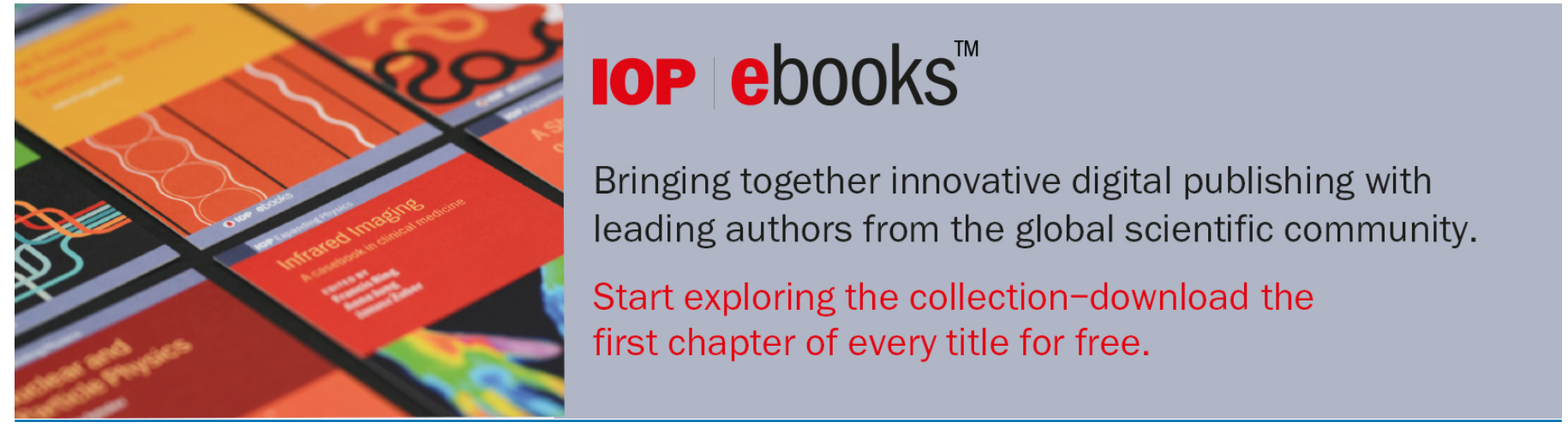

This content was downloaded from IP address 213.102 .95 .4 on 11/03/2021 at 12:38 


\title{
Multimillijoule terahertz radiation from laser interactions with microplasma waveguides
}

\author{
Ke Hu $\mathbb{D}^{-}$, Longqing Yi® and Tünde Fülöp \\ Department of Physics, Chalmers University of Technology, 41296 Gothenburg, Sweden \\ E-mail: longqing@chalmers.se
}

Received 30 September 2020, revised 17 December 2020

Accepted for publication 18 January 2021

Published 3 February 2021

\begin{abstract}
When a relativistic, femtosecond laser pulse enters a waveguide, the pulse energy is coupled into waveguide optical modes. The longitudinal laser field effectively accelerates electrons along the axis of the channel, while the asymmetric transverse electromagnetic fields tend to expel fast electrons radially outwards. At the exit of the waveguide, the $\sim \mathrm{nC}, \sim 10 \mathrm{MeV}$ electron bunch converts its energy to a $\sim 10 \mathrm{~mJ}$ terahertz $(\mathrm{THz})$ laser pulse through coherent diffraction radiation. In this paper, we present $3 \mathrm{D}$ particle-in-cell simulations and theoretical analyses of the aforementioned interaction process. We investigate the process of longitudinal acceleration and radial expulsion of fast electrons, as well as the dependence of the properties of the resulting $\mathrm{THz}$ radiation on laser and plasma parameters and the effects of the preplasma. The simulation results indicate that the conversion efficiency of energy can be over $5 \%$ if the waveguide length is optimal and a high contrast pump laser is used. These results guide the design of more intense and powerful $\mathrm{THz}$ sources.
\end{abstract}

Keywords: multimillijoule terahertz radiation, microplasma waveguide, electron acceleration, laser-plasma interaction

(Some figures may appear in colour only in the online journal)

\section{Introduction}

During the last decade, terahertz (THz) sources based on laserplasma interactions have attracted considerable attention due to their potential to produce $\mathrm{GV} \mathrm{cm} \mathrm{cm}^{-1}$, $\mathrm{mJ}$ level $\mathrm{THz}$ radiation. Such powerful $\mathrm{THz}$ sources would open up new regimes to investigate/manipulate physical systems across a broad range of research areas, ranging from biology to astrophysics [1-4]. Laser-driven underdense plasmas usually deliver a fewmicrojoule $\mathrm{THz}$ radiation in experiments and the $\mathrm{THz}$ energy saturates with increasing pump laser intensity [5-8]. However, particle-in-cell (PIC) simulations have predicted that

\footnotetext{
Original Content from this work may be used under the terms of the Creative Commons Attribution 4.0 licence. Any the title of the work, journal citation and DOI.
}

$\sim 1 \mathrm{GV} \mathrm{cm}^{-1}, \sim 1 \mathrm{~mJ} \mathrm{THz}$ pulses can be obtained by adopting a tailored pump laser [9] or by obliquely irradiating an underdense plasma slab with sub-100 $\mu \mathrm{m}$ thickness [10]. Employing laser-solid interactions appears even more promising [11-13]. As a solid foil is irradiated by a pump laser, coherent transition radiation in the $\mathrm{THz}$ range is emitted in the backward and forward directions, when the accelerated electrons pass through the front and rear surfaces of the target, respectively. Forward $\mathrm{THz}$ pulses with energy over $10 \mathrm{~mJ}$ have been experimentally demonstrated by Liao et al [14], with conversion efficiency at the level of $0.1 \%$.

The main factor in shaping the properties of coherent transition radiation from laser-driven solid targets is the quality of the electron beam $[15,16]$. The radiation is coherent if the bunch length is shorter than the radiated wavelength of interest, and the $\mathrm{THz}$ energy improves strongly with decreasing beam divergence. In the coherent regime, the radiated energy is proportional to the square of beam charge 
[17]. Therefore, an electron beam with high charge, high energy and small divergence is required for generating powerful $\mathrm{THz}$ radiation. For solid foil targets, the electron beam usually suffers from large divergence, and this prevents scaling the scheme toward higher THz energies [18].

In order to acquire high-quality electron beams and highenergy $\mathrm{THz}$ radiation, schemes utilizing micro-structured targets have been proposed. 3D PIC simulations demonstrate strong electron emission at the micro-scaled target edge, which leads to a $\mathrm{THz}$ energy of over $10 \mathrm{~mJ}$ with a $1 \mathrm{~J}$ pump laser [19]. Another study employs solid foil targets covered with aligned nanorod arrays in experiments; the resulting efficiency is enhanced by an order of magnitude compared to a solid foil target [20].

Among these structured targets is the microplasma waveguide (MPW); not only does it suppress the transverse diffraction of the pump laser, but it also enhances the longitudinal acceleration field [21, 22]. Such targets have already shown their potential in electron acceleration $[23,24]$, x-ray generation $[25,26]$, production of ion beams $[27,28]$ and manipulation of relativistic laser pulses [29]. In our previous work, simulations show that high charge $(\sim 10 \mathrm{nC})$, high energy $(\sim 100 \mathrm{MeV})$ and well-collimated $\left(10^{\circ}\right)$ electron bunches can be produced and accelerated by the transverse magnetic modes [30]. Their energies are converted to strong $\mathrm{THz}$ emission through coherent diffraction radiation (CDR) when they exit the MPW. Although that study has shown that an efficiency over $1 \%$ can be realized, even more powerful $\mathrm{THz}$ output can be reached by target optimization. The goal of the present paper is to optimize the laser and target parameters for highly efficient $\mathrm{THz}$ generation. This will be done by investigating the dynamics of fast electrons inside an MPW, in particular the effects of several target parameters, including target length and preplasma scale length, by means of 3D PIC simulations and analytic theory. It is found that the efficiency can be over $5 \%$ when the MPW length is optimal and the preplasma scale length is small.

The paper is organized as follows: section 2 introduces the optical modes and electron dynamics inside an MPW. Section 3 discusses properties of $\mathrm{THz}$ radiation as well as its dependence on laser-plasma parameters. In section 4 , the effect of preplasma is studied. At last, a brief summary is given in section 5.

\section{Electron dynamics}

The setup of a laser-MPW THz source is shown in figure 1(a). A linearly polarized laser pulse is tightly focused onto the entrance of a cylindrical waveguide along the $x$ axis, from the left. The electrons get accelerated inside the MPW and finally convert their energy to a relativistic half-cycle $\mathrm{THz}$ radiation pulse when leaving the channel. The interaction process is explored using the 3D PIC code EPOCH [31]. The dimensions of the simulation box are $x \times y \times z=80 \mu \mathrm{m} \times 100 \mu \mathrm{m}$ $\times 100 \mu \mathrm{m}$ with grid steps $d x \times d y \times d z=0.05 \mu \mathrm{m} \times 0.1 \mu \mathrm{m} \times$ $0.1 \mu \mathrm{m}$. The pump laser pulse has a temporal FWHM duration of $T_{0}=35 \mathrm{fs}$ and normalized amplitude of $a_{0}=e E_{0} / m c \omega_{0}=$
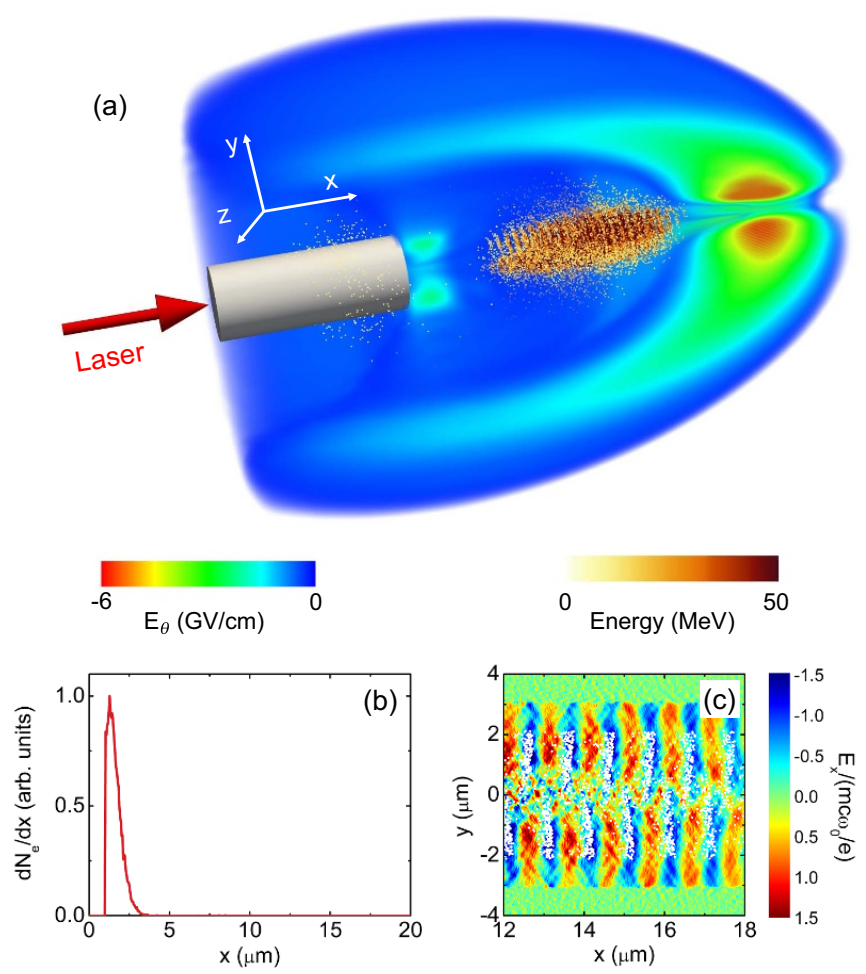

Figure 1. (a) 3D schematic setup of the $\mathrm{THz}$ radiation source based on an MPW. The orange dots are fast electrons (with relativistic gamma factor $\gamma>10$ ) at simulation time $t=200 \mathrm{fs}$ and the color represents their energies. The polar component of the $\mathrm{THz}$ field $E_{\theta}$ (frequency $f<60 \mathrm{THz}$ ) at $t=267 \mathrm{fs}$ is shown with rainbow colorscale, where a quarter is removed to display the intensity inside. (b) The distribution of the initial longitudinal positions of the electrons shown in (a). (c) Cross section of the longitudinal electric field inside the MPW at $t=107 \mathrm{fs}$ in the $x-y$ plane at where $z=0 \mu \mathrm{m}$. White dots represent projections of the positions of fast electrons on the $x-y$ plane.

10 , where $c$ is the speed of light, $m$ is the electron mass, $e$ is the unit charge, $\lambda_{0}=1 \mu \mathrm{m}$ is the laser wavelength and $\omega_{0}=2 \pi c / \lambda_{0}$ is the angular frequency. The spot size of the pulse is $w_{0}=3 \mu \mathrm{m}$. The MPW target has a length of $L=20 \mu \mathrm{m}$ and a density of $n_{0}=15 n_{c}$, where $n_{c}=\epsilon_{0} m \omega_{0}^{2} / e^{2}$ is the critical density. Its wall thickness and inner radius are $5 \mu \mathrm{m}$ and $r_{c}=3 \mu \mathrm{m}$, respectively. The entrance of the MPW is placed at $x=1 \mu \mathrm{m}$.

Figure 1(a) presents the $40.2 \mathrm{~mJ} \mathrm{THz}$ radiation pulse shown with rainbow color scale. The electric fields are considered in spherical coordinates with the origin at the exit of the MPW. The polar component $E_{\theta}$ contains $97 \%$ of the THz energy, which means that the $\mathrm{THz}$ emission is dominantly radially polarized. The peak electric amplitude reaches $7 \mathrm{GV} \mathrm{cm}^{-1}$, indicating that the $\mathrm{THz}$ pulse is relativistic. The optical-to$\mathrm{THz}$ conversion efficiency is $6.7 \%$, much higher than can be achieved by any other state-of-art laser-plasma $\mathrm{THz}$ source.

Strong $\mathrm{THz}$ radiation is emitted when the energetic electron bunch, represented by the orange dots in figure 1(a), leaves the channel. We track those electrons and plot the distribution of their initial locations along the $x$ direction in figure 1(b). We note that almost all these electrons originate from the 
vicinity of the entrance of MPW. This can be attributed to the strong diffracted laser light generated during the violent impact of the laser and onto the MPW front surface. Such diffraction-induced injection is the dominant mechanism for electron injection as long as $r_{c} \leqslant w_{0}$. In the next section we will show that the $\mathrm{THz}$ radiation under this condition is much more powerful than that in the case of $r_{c}>w_{0}$. Since our main interest is high-energy $\mathrm{THz}$ radiation, in the following, we focus on the interaction when $r_{c} \leqslant w_{0}$ and assume all fast electrons are to be injected at the MPW entrance.

We then proceed to discussing the propagation of the pump laser inside an MPW. Normally, many optical modes are excited simultaneously, and their intensities mainly depend on the waveguide radius. For the micro-scale waveguide used in our scheme, most of the laser energy is coupled into the fundamental waveguide mode and higher modes can be ignored [26], which effectively simplifies our analysis. Considering the shape of the target, it is appropriate to express the electromagnetic fields in cylindrical coordinates $(x, r, \phi)$ :

$$
\begin{gathered}
E_{x}=E_{0} \frac{k_{t}}{k} J_{1}\left(k_{t} r\right) \sin (\phi) \cos (\Phi) \\
B_{x}=-B_{0} \frac{k_{t}}{k} J_{1}\left(k_{t} r\right) \cos (\phi) \cos (\Phi) \\
E_{r}=-\frac{E_{0}}{2}\left[\frac{k+k_{x}}{k_{t}} J_{0}\left(k_{t} r\right)+\frac{k-k_{x}}{k_{t}} J_{2}\left(k_{t} r\right)\right] \sin (\phi) \sin (\Phi) \\
B_{r}=\frac{B_{0}}{2}\left[\frac{k+k_{x}}{k_{t}} J_{0}\left(k_{t} r\right)-\frac{k-k_{x}}{k_{t}} J_{2}\left(k_{t} r\right)\right] \cos (\phi) \sin (\Phi) \\
E_{\phi}=-\frac{E_{0}}{2}\left[\frac{k+k_{x}}{k_{t}} J_{0}\left(k_{t} r\right)+\frac{k-k_{x}}{k_{t}} J_{2}\left(k_{t} r\right)\right] \cos (\phi) \sin (\Phi) \\
B_{\phi}=-\frac{B_{0}}{2}\left[\frac{k+k_{x}}{k_{t}} J_{0}\left(k_{t} r\right)-\frac{k-k_{x}}{k_{t}} J_{2}\left(k_{t} r\right)\right] \sin (\phi) \sin (\Phi)
\end{gathered}
$$

where $E_{0}$ and $B_{0}$ are the amplitude of the electric and magnetic components of the pump laser, respectively. $k=2 \pi / \lambda_{0}$ is the wave number in vacuum and $\Phi$ is the phase within the laser pulse. $J_{\alpha}(x)$ denotes the Bessel function of the first kind of order $\alpha . k_{x}$ and $k_{t}$ are the longitudinal and transverse components of the wave number inside the MPW, respectively, which satisfy $k_{t}=x_{1} / r_{c}$ and $k_{t}^{2}+k_{x}^{2}=k^{2} . x_{1}$ is a parameter obtained by numerically solving an eigenvalue equation [22]. For the interaction geometry defined here, $x_{1}=2.5$ can be considered as a constant [26].

Structures of the longitudinal electric field $E_{x}$ in the $x-y$ plane are presented in figure 1(c). The amplitude of the acceleration gradient is $4 \mathrm{TV} \mathrm{m}^{-1}$, much higher than the longitudinal field of the pump laser in vacuum. Even though fast electrons acquire most of their energy from longitudinal acceleration, we note that only a small amount of laser energy is coupled to the longitudinal fields, namely $E_{x}^{2} \ll\left(E_{r}^{2}+E_{\phi}^{2}\right)$.
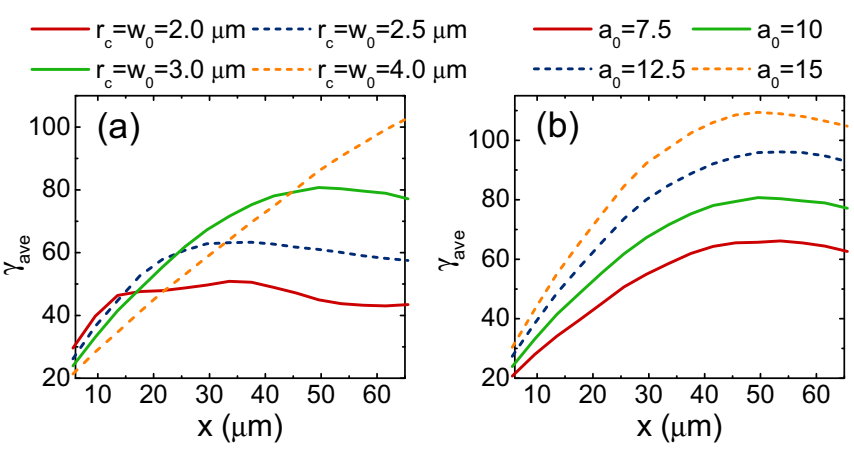

Figure 2. Average $\gamma$ of IFEs plotted against propagation distance of the pump laser for different $r_{c}$ (a) and $a_{0}$ (b). $a_{0}=10$ is fixed in (a), channel inner radius $r_{c}=3 \mu \mathrm{m}$ is fixed in (b) and laser spot size $w_{0}=r_{c}$ is satisfied in both panels.

One important feature of $E_{x}$ is that its phase velocity is superluminal [22], which means that energetic electrons undergo first an acceleration stage when they are in desirable phases (see figure 1(c)), then a deceleration stage after the dephasing effect takes place. The phase varies from $\Phi=0$ to $\Phi=\pi$ at the acceleration stage. The maximum acceleration distance $L_{a c c}$ satisfies [30]

$$
L_{\mathrm{acc}} \propto r_{c}^{2}
$$

From equation (1), we can deduce that the peak acceleration gradient is proportional to $a_{0} / r_{c}$. Therefore the maximum relativistic gamma factor fulfills

$$
\gamma_{\max } \propto r_{c} a_{0}
$$

Next, we show a series of simulations, in which the laser waist size equals the channel inner radius, ranging from 2.0 to $4.0 \mu \mathrm{m}$. A few-cycle pump laser $\left(T_{0}=10 \mathrm{fs}\right)$ is adopted in order to lower the length of the electron bunch, making it easier to distinguish the acceleration or deceleration stage. As soon as the laser enters the waveguide, excited electrons $(\gamma>3)$ are selected out and their dynamics are tracked every four laser cycles during the simulation. At each moment we exclude escaping electrons $\left(r>r_{c}\right)$, and only consider energetic electrons $(\gamma>10)$ that are inside the tube, which we call internal fast electrons (IFEs) hereafter.

The average gamma factor of IFEs versus propagation distance of the pump laser, for different $w_{0}$ and $r_{c}$ are plotted in figure 2(a). At the acceleration stage, the average electron energy increases rapidly for small $r_{c}$, since the acceleration field is inversely proportional to MPW radius. However, the violent acceleration phase only lasts for a short time and the saturation energy is below $25 \mathrm{MeV}$ when $r_{c}=2 \mu \mathrm{m}$. On the contrary, when the channel radius is larger, the acceleration is weaker but lasts for a longer time, finally leads to a higher saturation energy.

Similarly, in figure 2(b), we present the effect of laser amplitude on the acceleration process, in the case of a fixed channel radius $r_{c}=3 \mu \mathrm{m}$. The electron energies reach their maximum values at almost the same time, since the acceleration length 


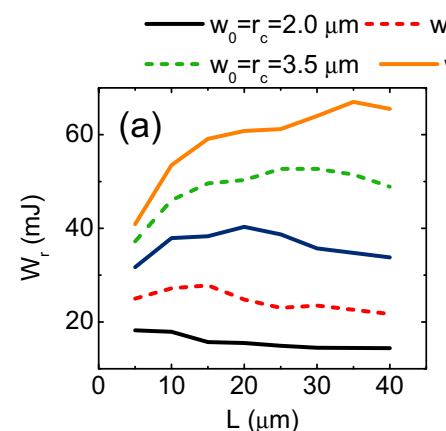

$$
w_{0}=r_{c}=2.5 \mu \mathrm{m} \longrightarrow w_{0}=r_{c}=3.0 \mu \mathrm{m}
$$

$$
\mathrm{w}_{0}=\mathrm{r}_{\mathrm{c}}=4.0 \mu \mathrm{m}
$$
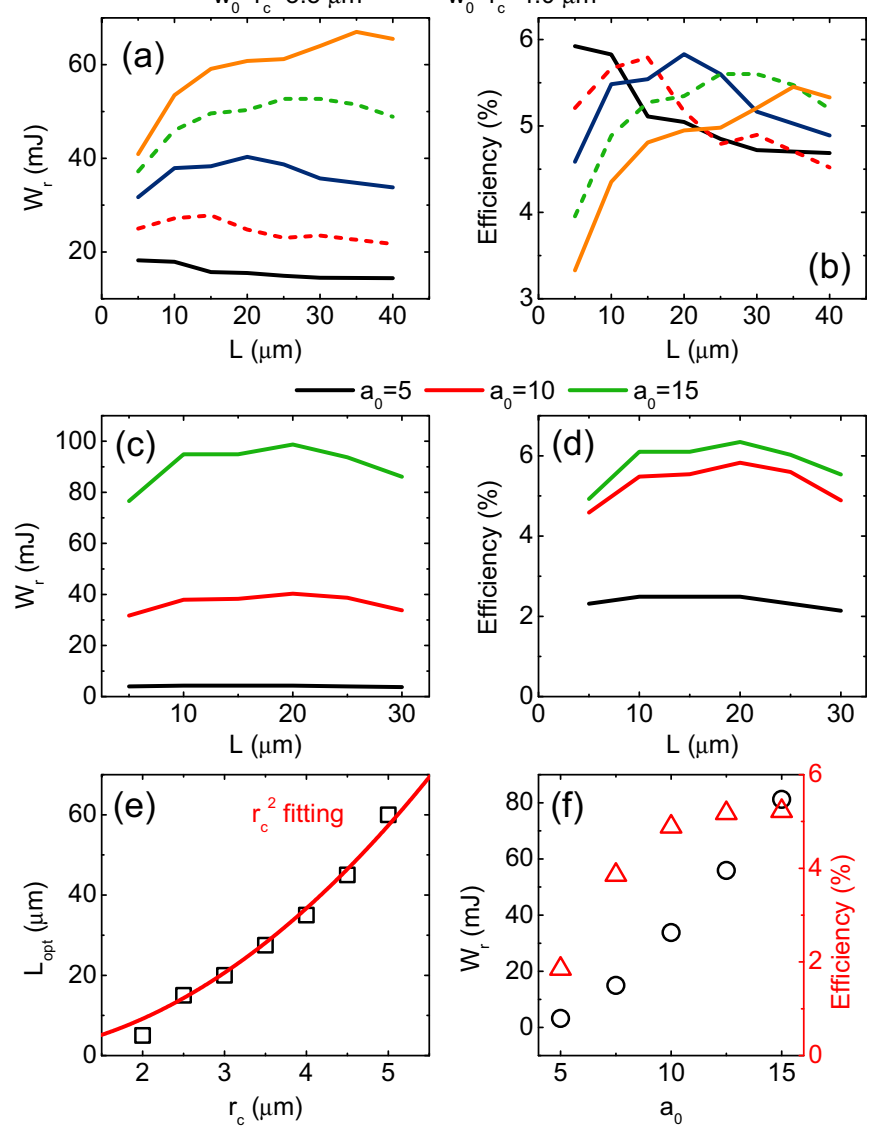

Figure 3. Fraction of radially escaping electrons plotted against propagation distance of the pump laser for different $r_{c}$ (a) and $a_{0}$ (b). $a_{0}=10$ is fixed in (a), $r_{c}=3 \mu \mathrm{m}$ is fixed in (b), and $w_{0}=r_{c}$ is satisfied in all panels.

is not related to the pump laser amplitude (equation (7)). Furthermore, the saturation energy increases with laser amplitude, in agreement with equation (8).

We now proceed to discuss the effect of transverse optical modes on electron dynamics. Using equations (3)-(6), the transverse force acting on the IFEs can be derived as

$$
F_{\perp}=e \sqrt{\left(E_{r}-B_{\phi}\right)^{2}+\left(E_{\phi}-B_{r}\right)^{2}} \propto \frac{a_{0}}{r_{c}^{2}} \sin (\Phi) .
$$

The force can lead to a maximum transverse displacement $r_{\max }$, that scales as

$$
r_{\max } \propto a_{0} r_{c}^{2}
$$

At the same time, the relative phase varies from $\Phi=0$ to $\Phi=\pi / 2$, which means that the IFEs have passed approximately half of the maximum acceleration distance in the $x$ direction. For small channel radius, the maximum acceleration distance is short, hence a large fraction of fast electrons tend to be scattered and escape earlier. Those escaping electrons transmit into the target bulk through inner boundaries of the MPW before reaching the channel exit, thus making a negligible contribution to $\mathrm{THz}$ emission.
Figures 3(a) and (b) show the fraction of escaping electrons among the energetic ones versus the pump laser's propagation distance for different channel radii and laser amplitudes, respectively. Electrons escape from the channel fast when the channel radius is small, same as what we predicted above. Since the maximum acceleration distance is not related to the laser amplitude, the rates of reduction in electron charge are approximately equal for different $a_{0}$.

\section{Terahertz radiation}

The radiated $\mathrm{THz}$ energy scales as the square of the beam charge that leaves the waveguide. The electron beam consists of electrons that fulfill the following two conditions: (i) have high enough energies to escape through the exit; (ii) are not expelled from the channel radially before arriving in the exit. A short MPW is not favorable for the pump laser to deposit its energy to more IFEs, whereas in a long target, more fast electrons will escape during the long propagation. Therefore the MPW has an optimal length $L_{\mathrm{opt}}$ at which the number and energy of IFEs are most advantageous to the emission of $\mathrm{THz}$ radiation. The scattering angle of the IFEs can be roughly estimated by $\tan (\Theta) \approx r_{\max } / L_{\text {acc }}$. Therefore, using equations (7) and (10), we obtain

$$
L_{\mathrm{opt}}=\frac{r_{c}}{\tan (\Theta)} \propto r_{c}^{2}
$$

A series of 3D PIC simulations are conducted to investigate the optimal MPW length. The laser pulse duration is set to $T_{0}=35 \mathrm{fs}$. Since the THz frequency depends on the length of the electron bunch and it is close to the pump laser duration, $T_{0}=35$ fs leads to a central THz frequency of about $3 \mathrm{THz}$. We introduce preplasma at the inner surface of the MPW, in order to better simulate the interaction in realistic conditions. The preplasma has a density profile $n(r)=n_{0} \exp \left[-\left(r-r_{0}\right)^{2} / \sigma_{0}^{2}\right]$, where $\sigma_{0}$ is the scale length and $r_{0}$ is the inner radius. Here $r_{c}$ refers to the effective channel radius, which satisfies $n\left(r_{c}\right)=$ $n_{c}$. Under this condition, the previous equations are valid in the presence of preplasma. Here, the scale length is set as $\sigma_{0}=0.2 \mu \mathrm{m}$; a discussion on its effect can be found in the next section.

Figures 4(a) and (b) show the $\mathrm{THz}$ energy and conversion efficiency against the length of MPW for different MPW radius $r_{c}$, respectively. It can be observed that the energy of $\mathrm{THz}$ radiation is typically over $10 \mathrm{~mJ}$. As the MPW length is increased, the $\mathrm{THz}$ energy first increases then reduces in every case except for $r_{c}=2.0 \mu \mathrm{m}$. The reason is that when the tube radius is small, the effect of expulsion is so severe that it has a more significant impact than the effect of acceleration in a very early stage. Figure 4(e) shows the relationship between optimal MPW length and channel radius. The dependence of $L_{\mathrm{opt}}$ on $r_{c}$ has a squared scaling as predicted by equation (11). From figure 4(b), one may also notice that as the length of MPW increases, the dependence of conversion efficiency on the tube radius becomes less and less important. This is because in a short MPW, only a small fraction 

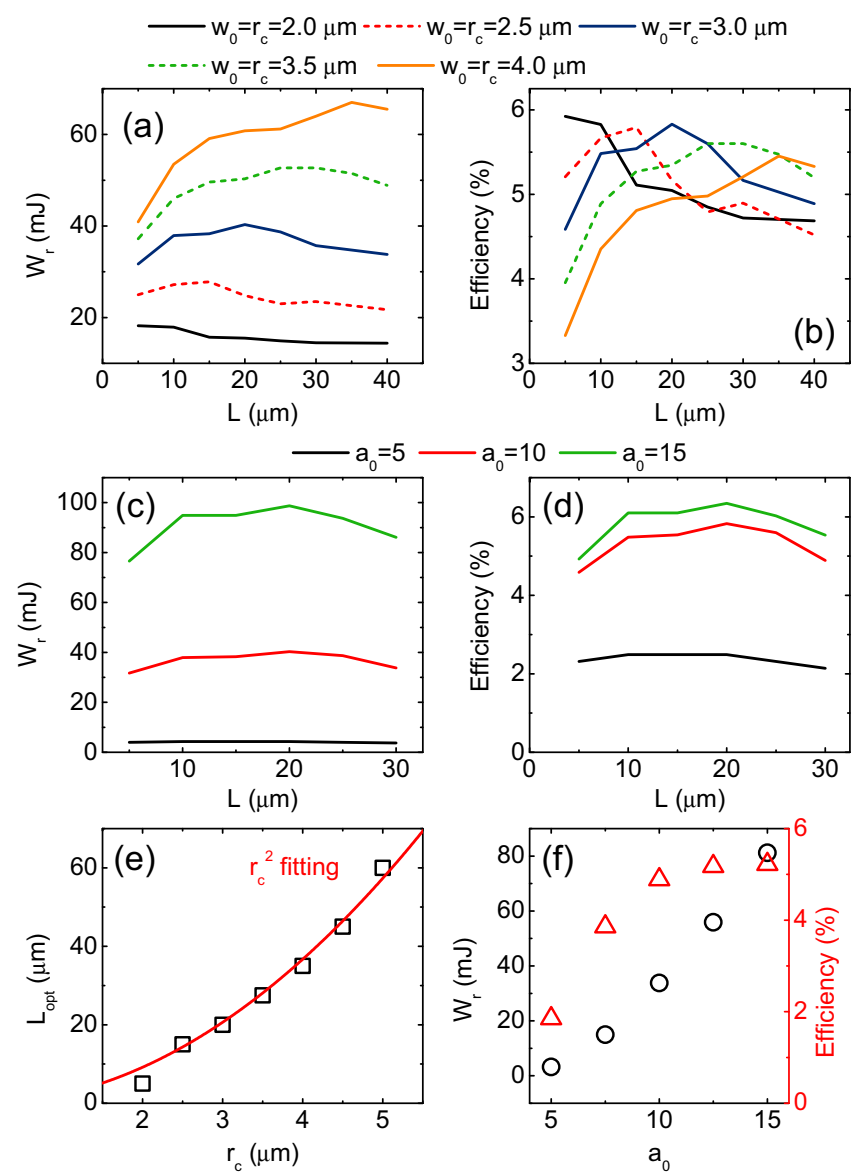

Figure 4. Dependence of THz energy ((a) and (c)) and conversion efficiency of energy ((b) and (d)) on the length of MPW for different $r_{c}\left((\mathrm{a})\right.$ and (b)) and $a_{0}\left((\mathrm{c})\right.$ and (d)). $a_{0}=10$ is fixed in (a) and (b), while $r_{c}=w_{0}=3 \mu \mathrm{m}$ is fixed in (c) and (d). (e) The optimal length of MPWs vs $r_{c}$ for fixed $a_{0}=10$. (f) Peak THz radiation energy and conversion efficiency vs varying laser amplitude $a_{0}$ for fixed $r_{c}=3 \mu \mathrm{m} . w_{0}=r_{c}$ is satisfied in (e) and (f).

of the drive laser near the wall can interact with the injected electrons near the entrance. This energy fraction, which is inversely proportional to $r_{c}$, predominately determines the overall efficiency. When the MPW is sufficiently long, there is enough time for the electrons to gain energy from the entire laser beam (see figures 2(a) and 3(a)), which results in similar efficiencies for different tube radius.

Similarly, in figures 4(c) and (d), we present the THz energy and conversion efficiency under different laser amplitudes. When the laser spot size and channel radius are fixed at $w_{0}=$ $r_{c}=3 \mu \mathrm{m}$, the optimal MPW length is the same for laser amplitudes ranging from $a_{0}=5-15$. From figure 4(c), we see that the peak $\mathrm{THz}$ energy reaches $99 \mathrm{~mJ}$ in the case of $a_{0}=15$ (corresponding to a pump laser energy of $1.6 \mathrm{~J}$ ). In addition, figure 4(f) illustrates that the peak efficiency increases with $a_{0}$ and finally saturates at about $6 \%$ when $a_{0}>10$. Keeping in mind that the energy spectrum of IFEs satisfies an approximately Maxwellian distribution, a small laser amplitude leads to a small saturation energy, thus many relatively low-energy electrons (distributed on the low-energy part of the spectrum) are not energetic enough to escape from the MPW.

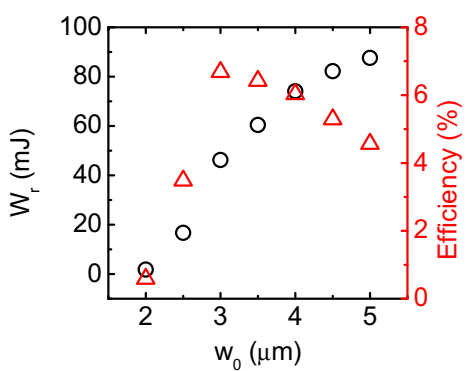

Figure 5. THz radiation energy and conversion efficiency as function of laser spot size $w_{0}$. The other parameters are $r_{c}=3 \mu \mathrm{m}$, $a_{0}=10$ and $L=20 \mu \mathrm{m}$.

This accounts for the rapidly dropping efficiency as $a_{0}$ is decreased below 10 .

Finally, figure 5 shows a scan of the $\mathrm{THz}$ energy and efficiency plotted against laser spot size $w_{0} . r_{c}=3 \mu \mathrm{m}$ remains unchanged to ensure the same optimal MPW length $L=20 \mu \mathrm{m}$ for all cases. The $\mathrm{THz}$ energy increases with laser spot size, while the efficiency reaches its maximum at $w_{0}=r_{c}$. Increasing $w_{0}$ leads to enhanced electron excitation at the entrance and more powerful electron acceleration inside the channel, thus helps for the boost of THz energy. However, for $w_{0}>r_{c}$, increasing $w_{0}$ means a smaller fraction of the laser that can make its way to the channel, which is responsible for the decrease of efficiency.

\section{Preplasma effects}

In relativistic laser interaction with micro-scale channel target, the preplasma condition, in particular the scale length of the density profile on the inner boundary, has a significant impact on the number and energy of fast electrons, as well as the energy of $\mathrm{THz}$ radiation. In figure 6(a), $\mathrm{THz}$ energy is plotted against the scale length $\sigma_{0}$ in the case of $a_{0}=10, L=20 \mu \mathrm{m}$, $r_{c}=w_{0}=3 \mu \mathrm{m}$. We note that the $\mathrm{THz}$ energy decreases with increasing preplasma scale length. When $\sigma_{0}=0.8 \mu \mathrm{m}$, the $\mathrm{THz}$ radiation has an energy of $18 \mathrm{~mJ}$, corresponding to about $25 \%$ of the case without preplasma.

The main reason for the detrimental effect of the preplasma is that the distribution of electromagnetic fields inside an MPW is modified. To illustrate this, we use 3D PIC simulations with higher resolution $(d x \times d y \times d z=0.02 \mu \mathrm{m} \times$ $0.04 \mu \mathrm{m} \times 0.04 \mu \mathrm{m})$, while the other parameters remain the same. Figures 6(b) and (c) present the distribution of acceleration gradient $E_{x}$ for $\sigma_{0}=0$ and $\sigma_{0}=0.8 \mu \mathrm{m}$, respectively. The snapshot was taken at the moment when the pump laser pulse has propagated a distance of $20 \mu \mathrm{m}$. It is evident that the electric field in figure 6(c) is greatly distorted and is no longer radially uniform, resulting in less IFEs that can be accelerated steadily for a sufficiently long time.

In essence, the distortion of optical modes can be attributed to the oscillation of inner waveguide surfaces, induced directly by the radial component of the laser field. Such oscillations are violent in the region of near-critical-density preplasmas $[32,33]$, which can be illustrated by a snapshot of density 

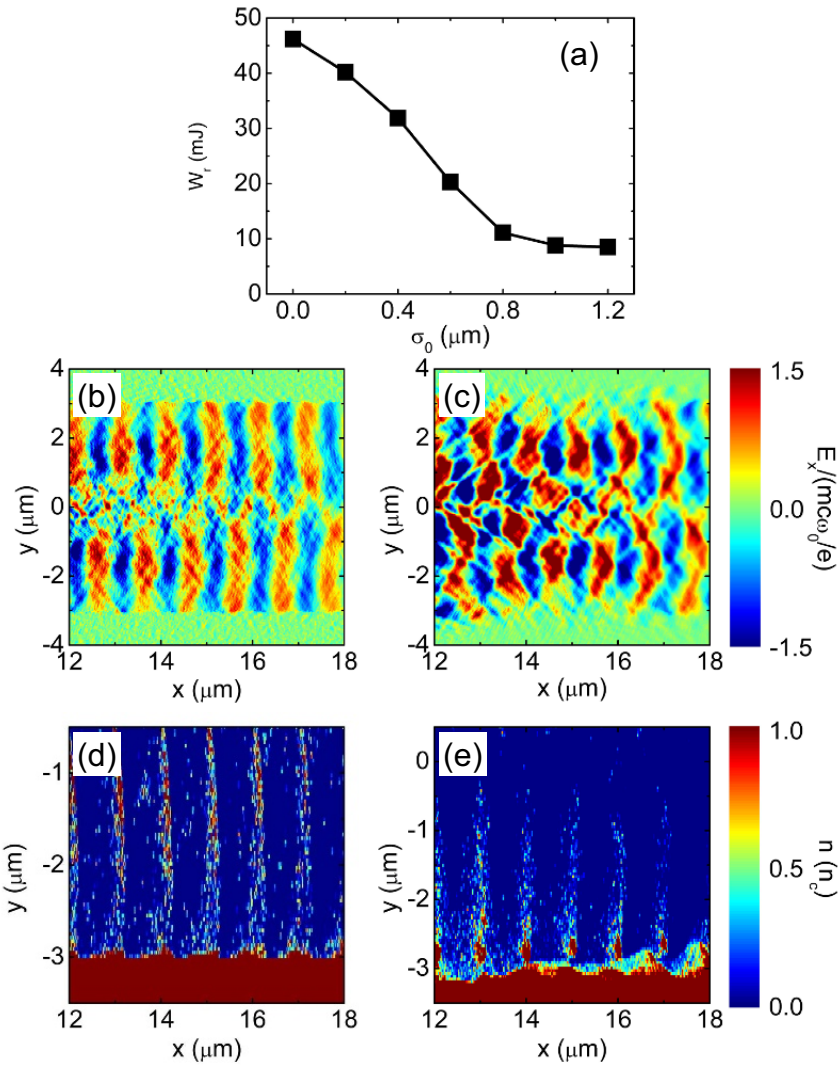

Figure 6. (a) $\mathrm{THz}$ radiation energy as a function of the preplasma density scale length. (b), (c) The distribution of longitudinal electric field inside the MPW at $t=200 \mathrm{fs}$ for $\sigma_{0}=0 \mu \mathrm{m}$ and $\sigma_{0}=0.8 \mu \mathrm{m}$, respectively. (d), (e) The density distribution of inner boundaries of MPWs under the same condition as in (b) and (c), respectively.

profile around the inner plasma surface when $\sigma_{0}=0.8 \mu \mathrm{m}$, plotted in figure 6(e). The amplitude of oscillation is so large that overdense electron clusters are extracted from the surface and then reinjected into the bulk plasma. In contrast, in figure 6(d), one sees mild density oscillations in the case of a step boundary of overdense plasma. Therefore, in order to obtain high THz energy, it is required to lower the scale length of preplasmas by using high contrast pump lasers.

\section{Conclusion}

We have studied a mechanism of relativistic $\mathrm{THz}$ radiation generation based on laser interaction with MPWs via CDR. The critical underlying physical processes involve acceleration and expulsion of fast electrons. The former can be attributed to powerful longitudinal component of the optical modes inside the MPW, while the latter is induced by a weak transverse force caused by the asymmetry in the transverse components of these optical modes. The two processes for different laser amplitudes and waveguide radii are investigated via a theoretical model and 3D PIC simulations, in order to find parameters that optimize the optical-to-THz conversion efficiency.

It is found that the optimal length is proportional to the square of MPW radius and is independent of laser amplitude. Typically, a THz radiation pulse at the order of a few tens of millijoule can be obtained from a joule level pump laser, corresponding to a peak efficiency over $6 \%$. In addition, it is found that preplasma is detrimental to $\mathrm{THz}$ generation due to the distorted distribution of optical modes.

Finally, we note that here we focus on MPW THz sources under ideal condition of normal on-axis incidence. The misalignment of the drive laser in the experiment would affect the conversion efficiency. However, state-of-art high-power laser facilities can reach a pointing stability on the order of $1 \mu \mathrm{rad}$ with an active control system [34], and the feasibility of laser-MPW interaction has been demonstrated experimentally [23]. Our simulations show that a small incident angle of $\sim 1$ mrad makes little difference compared with the ideal case under consideration. For oblique incidence with a much larger angle, the electromagnetic field in the MPW can no longer be described with standard waveguide modes. A detailed analysis is out of scope of the current work.

Our results directly assist the design of $\mathrm{MPW} \mathrm{THz}$ sources that can reach unprecedented conversion efficiencies among laser-plasma based approaches, and provide theoretical insights into the relevant microphysical processes.

\section{Acknowledgments}

The authors acknowledge fruitful discussions with I Pusztai. This work is supported by the Olle Engqvist Foundation and the European Research Council (ERC-2014-CoG Grant 647121). Simulations were performed on resources at Chalmers Centre for Computational Science and Engineering (C3SE) provided by the Swedish National Infrastructure for Computing (SNIC).

\section{ORCID iDs}

Ke Hu (D) https://orcid.org/0000-0001-5935-6464

Longqing Yi (D) https://orcid.org/0000-0003-2378-5480

Tünde Fülöp (D) https://orcid.org/0000-0002-5898-0393

\section{References}

[1] Hebling J A, Yeh K L, Hoffmann M C and Nelson K A 2008 IEEE J. Sel. Top. Quantum Electron. 14 345-53

[2] Pickwell E and Wallace V P 2006 J. Phys. D: Appl. Phys. 39 R301

[3] Siegel P H 2004 IEEE Trans. Microw. Theory Tech. 522438

[4] Globus T, Woolard D L, Khromova T, Crowe T W, Bykhovskaia M, Gelmont B L, Hesler J and Samuels A C 2003 J. Biol. Phys. 2989

[5] Leemans W P et al 2003 Phys. Rev. Lett. 91074802

[6] Clerici M et al 2013 Phys. Rev. Lett. 110253901

[7] Xie X, Dai J and Zhang X C 2006 Phys. Rev. Lett. 96075005

[8] Déchard J, Debayle A, Davoine X, Gremillet L and Bergé L 2018 Phys. Rev. Lett. 120144801

[9] Chen Z Y 2016 AIP Adv. 6065302

[10] Wu H C, Sheng Z M and Zhang J 2008 Phys. Rev. E 77046405

[11] Liao G Q et al 2016 Plasma Phys. Control. Fusion 59014039

[12] Ding W J and Sheng Z M 2016 Phys. Rev. E 93063204

[13] Liao G Q and Li Y T 2019 IEEE Trans. Plasma Sci. 473002

[14] Liao G Q et al 2019 Proc. Natl. Acad. Sci. USA 1163994 
[15] Schroeder C B, Esarey E, Tilborg J and Leemans W P 2004 Phys. Rev. E 69016501

[16] Ding W J, Li F Y, Weng S M, Bai P and Sheng Z M 2019 (arXiv:1902.04716)

[17] Zheng J, Tanaka K A, Miyakoshi T, Kitagawa Y, Kodama R, Kurahashi T and Yamanaka T 2003 Phys. Plasmas 102994

[18] Liao G Q et al 2016 Phys. Rev. Lett. 116205003

[19] Hu K and Yi L 2020 Phys. Rev. A 102023530

[20] Mondal S et al 2017 Sci. Rep. 740058

[21] Gong Z, Robinson A P L, Yan X Q and Arefiev A V 2019 Plasma Phys. Control. Fusion 61035012

[22] Shen H 1991 J. Appl. Phys. 696827

[23] Snyder J et al 2019 Phys. Plasmas 26033110

[24] Xiao K D et al 2016 Phys. Rev. E 93043207

[25] Yi L, Pukhov A, Luu-Thanh P and Shen B 2016 Phys. Rev. Lett. 116115001
[26] Yi L, Pukhov A and Shen B 2016 Phys. Plasmas 23073110

[27] Zou D B, Pukhov A, Yi L, Zhuo H B, Yu T P, Yin Y and Shao F Q 2015 Sci. Rep. 742666

[28] Kluge T et al 2012 New J. Phys. 14023038

[29] Ji L L, Snyder J, Pukhov A, Freeman R R and Akli K U 2016 Sci. Rep. 623256

[30] Yi L and Fülöp T 2019 Phys. Rev. Lett. 123094801

[31] Arber T D et al 2015 Plasma Phys. Control. Fusion 57113001

[32] Lichters R, Meyer-ter-Vehn J and Pukhov A 1996 Phys. Plasmas 33425

[33] Bulanov S V, Naumova N M and Pegoraro F 1994 Phys. Plasmas 1745

[34] Genoud G, Wojda F, Burza M, Persson A and Wahlström C-G 2013 Rev. Sci. Instrum. 82033102 